\title{
High-dose rate brachytherapy for local recurrent adenoid cystic carcinoma of the tongue base following postoperative external beam radiotherapy
}

\author{
SUN YOUNG LEE ${ }^{1,2}$, JUNG SOO KIM ${ }^{1,2}$ and HYOUNG CHEOL KWON ${ }^{1,2}$ \\ ${ }^{1}$ Department of Radiation Oncology, ${ }^{2}$ Institute of Clinical Medicine of Chonbuk National University-Biomedical \\ Research Institute, Chonbuk National University Hospital, Jeonju 561-712, Republic of Korea
}

Received October 2, 2015; Accepted January 20, 2016

DOI: $10.3892 / \operatorname{mco} .2016 .999$

\begin{abstract}
Adenoid cystic carcinoma is a rare neoplasm commonly originating from the minor salivary glands. The clinical findings typical of this tumor include slow growth, perineural invasion and high frequency of local recurrence. In this study, a patient presented with a tongue base lesion that was treated with surgical excision and additional postoperative external beam radiotherapy. However, local recurrence occurred 8 months after radiotherapy. If recurrence occurs after radiation therapy, total glossectomy should be considered. However, the patient refused re-operation and, considering the patient's age, brachytherapy was used to ensure organ preservation. Complete remission was achieved following brachytherapy, without serious side effects. There has been no progression of the lesion during a follow-up period of 2 years.
\end{abstract}

\section{Introduction}

Adenoid cystic carcinoma is a relatively rare epithelial tumor of the minor salivary glands that is clinically characterized by slow growth, high frequency of local recurrence and perineural invasion $(1,2)$. The most common localization is the parotid gland, followed by the submandibular and sublingual glands (2). The reported frequency of occurrence in the tongue is $19.8 \%$, with $85 \%$ occurring in the tongue base (3-5). Surgical excision with wide margins is the treatment of choice when the cancer metastasizes to the lymph nodes; postoperative radiotherapy is recommended for cases with positive surgical margins $(2,4)$. In this case study, an adenoid cystic carcinoma of the tongue base that recurred locally following postoperative radiation therapy, was successfully treated with brachytherapy.

Correspondence to: Professor Hyoung Cheol Kwon, Department of Radiation Oncology, Chonbuk National University Hospital, 20 Geonji-Ro, Deokjin-Gu, Jeonju-Si, Jeollabuk-do, Jeonju 561-712, Republic of Korea

E-mail: hckwon@jbnu.ac.kr

Key words: adenoid cystic carcinoma, local recurrence after postoperative radiotherapy, brachytherapy

\section{Case report}

A 50-year-old woman complained of a sore throat and discomfort during swallowing. An intraoral examination by laryngoscope revealed a whitish yellow cystic mass at the base of the tongue. The histopathological analysis revealed an adenoid cystic carcinoma; on magnetic resonance imaging (MRI), the tumor was sized $25 \times 25 \times 30 \mathrm{~mm}$ and invaded the intrinsic muscle of the left posteroinferior tongue. The tumor was staged as cT4NOM0. Following wide local excision, three-dimensional conformal radiotherapy was performed at a total dose of $56 \mathrm{~Gy}$. Complete remission was obtained following radiotherapy; however, during follow-up, local recurrence was observed at the tongue base on visual inspection and laryngoscopic examination (Fig. 1). The suspected recurrence was investigated by MRI, revealing a primary tumor confined to the lateral tongue base. After detecting the tumor recurrence, a biopsy was performed, confirming that the recurrence of the adenoid cystic carcinoma was confined to the mucosa. Considering the patient's age, brachytherapy was performed in the area surrounding the tumor to preserve the tongue function.

Brachytherapy was administered using a computer-assisted high-dose rate remote afterloading dose delivery system (MicroSelectron; Nucletron-Elekta, Veenendaal, The Netherlands) utilizing a single ${ }^{192} \mathrm{Ir}$ source with an activity at installation of $\sim 370 \mathrm{GBq}$. A total dose of $30 \mathrm{~Gy}$ (biologically effective dose, 36.0 Gy) was administered twice per week in 3-Gy fractions. The brachytherapy target volume was determined individually for this patient using the MRI findings. The target volume encompassed the recurrent tumor as visualized by MRI and endoscopic examination, as well as a 1.5-cm margin proximal and distal to the tumor. The image with a dummy source, which was taken during a conventional simulation, was transferred to the intraluminal brachytherapy catheter. The geometrically optimized dose was generated using the Plato BPS v14.2.6 planning system (Nucletron-Elekta) (Fig. 2). Considering the lesions, we used the mold with a left lateral position. The velum stimulation often results in severe gag reflex during treatment. In this case, the uvula was anesthetized using lidocaine spray prior to treatment (Fig. 3). Three months after brachytherapy, the patient underwent MRI and laryngoscopic examination; the 

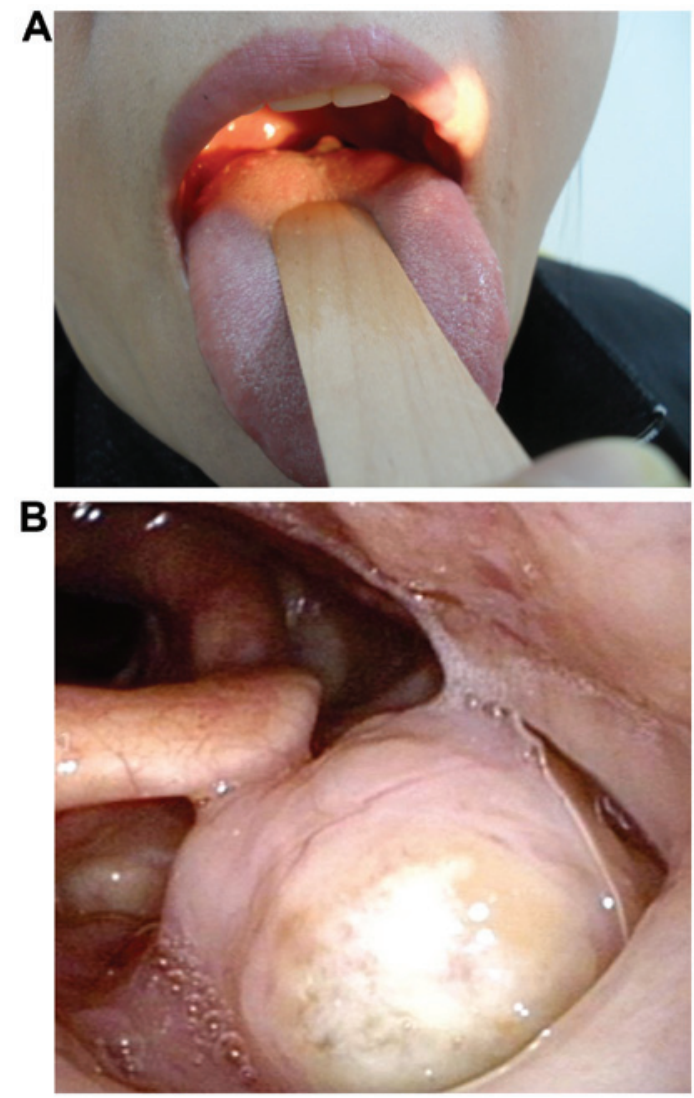

Figure 1. (A and B) A nodule protruding from the root of the tongue was identified on visual inspection and laryngoscopic examination; the nodule was the same color as the adjacent oral mucosa.

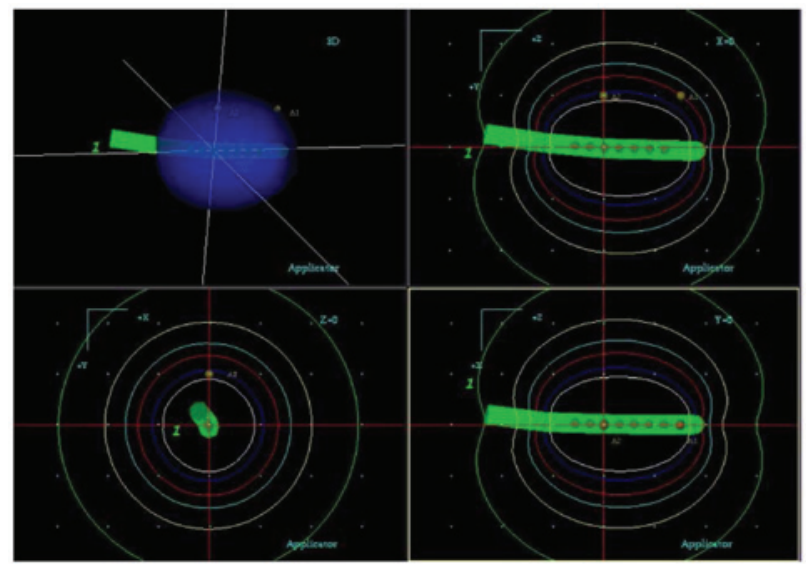

Figure 2. The geometrically optimized radiation dose was generated using the Nucletron-Elekta Plato BPS v14.2.6 planning system. A total dose of 30 Gy was administered twice per week in 3-Gy fractions.

mass was not identified (Fig. 4). In addition, no cancer cells were detected at the site of the previous tumor biopsy. During treatment, acute grade 2 radiation mucositis occurred, which subsided within 3 week after treatment completion. Chronic side effects did not occur. There has been no tumor recurrence during the 2 years of follow-up.

This study was approved by the Ethics Committee of Chonbuk National University Hospital Institutional Review Board and was performed in compliance with the guidelines

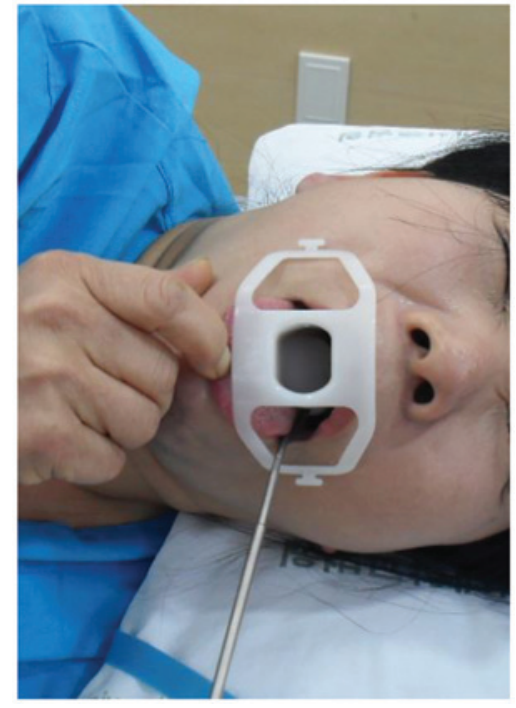

Figure 3. Considering the lesions, we used the mold in the left lateral position. Velum stimulation often leads to severe gag reflex during treatment. In this case, the uvula was anesthetized using lidocaine spray prior to treatment.

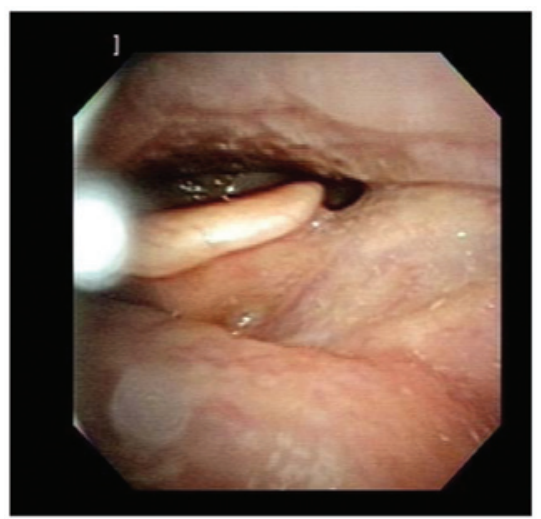

Figure 4. Following brachytherapy, the patient achieved complete remission according to the laryngoscopic examination.

of the Helsinki Declaration of 1975, as revised in 2008. The patient provided written informed consent and the clinical data were anonymized for statistical work-up.

\section{Discussion}

Adenoid cystic carcinomas constitute $29.6 \%$ of all minor salivary gland tumors, and their occurrence in the tongue is extremely rare $(4.4 \%)(3,5,6)$. The most common treatment for adenoid cystic carcinoma is wide surgical excision (4). Alternative treatment options include radiotherapy, chemotherapy and combination therapy (surgical excision and radiotherapy, or radiotherapy and chemotherapy) $(5,6)$. High recurrence rates occur following surgical resection of the lesion, but complete resection is difficult, as the resection margin is often positive due to the presence of a tumor cell $(5,7)$. Therefore, even when surgical resection is performed, postoperative radiation therapy should be considered in the majority of the patients for conservative treatment. In addition, adenoid cystic carcinoma exhibits strong neurotropism and tends to invade nerves $(6,8)$. Consequently, surgical excision 
or radiotherapy alone may fail to eliminate relapse in the surgical margins $(4,6)$. Distant metastasis is also occasionally observed in the cervical lymph nodes, lungs, bone and brain. In our patient, external beam radiotherapy following surgery made it necessary to limit the dosage of additional external beam radiation to the peripheral organs despite re-recurrence, making treatment difficult $(9,10)$. Although total glossectomy is an option following postoperative radiotherapy, due to the age of the patient and the desire for long-term, postoperative functional recovery, mold brachytherapy was performed locally. The additional radiation therapy doses were a limitation, but since no serious adverse events occurred, we were able to end treatment after observing complete disappearance of the lesions. There was no tumor recurrence during follow-up. However, adenoid cystic carcinoma may be associated with long-term local recurrence and distant metastasis, indicating the need for additional follow-up. If local recurrence occurs at the primary site following long-term disease control, brachytherapy should be considered.

Adenoid cystic carcinoma recurrence following surgery is commonly reported after conservative treatment is applied. However, when recurrence occurs after radiation therapy, the most common treatment is complete resection of the tongue. However, when preservation of organ function is desired, brachytherapy should be considered. In the present case, complete remission was achieved in a patient with recurrent adenoid cystic carcinoma following brachytherapy, without serious side effects.

\section{Acknowledgements}

The present study was supported by a fund from the Institute of Clinical Medicine of Chonbuk National University-Biomedical
Research Institute, Chonbuk National University Hospital, Jeonju, Republic of Korea.

\section{References}

1. Raux-Rakotomalala F, Houliat T, Martel J, Stoll D, Bébéar JP and Darrouzet V: Adenoid cystic carcinoma of head and neck: A review of 30 cases. Rev Laryngol Otol Rhinol (Bord) 124: 235-241, 2003 (In French).

2. Iseli T, Karnell LH, Graham SM, Funk GF, Buatti JM, Gupta AK, Robinson RA and Hoffman HT: Role of radiotherapy in adenoid cystic carcinoma of the head and neck. J Laryngol Otol 123: 1137-1144, 2009.

3. Namazie A, Alavi S, Abemayor E, Calcaterra TC and Blackwell KE: Adenoid cystic carcinoma of the base of the tongue. Ann Otol Rhinol Laryngol 110: 248-253, 2001.

4. Soares EC, Carreiro Filho FP, Costa FW, Vieira AC and Alves AP: Adenoid cystic carcinoma of the tongue: Case report and literature review. Med Oral Patol Oral Cir Bucal 13: E475-E478, 2008.

5. Traiantafillidou K, Dimitrakopoulous J, Iprdanidis F and Koufogiannis D: Management of adenoid cystic carcinoma of minor salivary glands. J Oral Maxillofas Surg 64: 1114-1120, 2006.

6. Iyer NG, Kim L, Nixon IJ, Palmer F, Kraus D, Shaha AR, Shah JP, Patel SG and Ganly I: Factors predicting outcome in malignant minor salivary gland tumors of the oropharynx. Arch Otoloryngol Head and Neck Surg 136: 1240-1247, 2010.

7. Bradely PJ: Adenoid cystic carcinoma of the head and neck: A review. Curr Opin Otolaryngol Head Neck Surg 12: 127-132, 2004.

8. Carrasco Ortiz D and Aldape Barrios B: Adenoid cystic carcinoma of the dorsum of the tongue: Presentation of a case. Med Oral Patol Oral Cir Bucal 11: E417-E420, 2006 (In English, Spanish).

9. Jaber MA: Intraoral minor salivary gland tumors: A review of 75 cases in a Libyan population. Int J Oral Maxillofac Surg 35: 150-154, 2006.

10. Wang D, Li Y, He H, Liu L, Wu L and He Z: Intraoral minor salivary gland tumors in a Chinese population: A retrospective study on 737 cases. Oral Surg Oral Med Oral Pathol Oral Radiol Endod 104: 94-100, 2007. 